\title{
A Common Coordinates/Heading Direction Generation Method for a Robot Swarm with Only RSSI-Based Ranging
}

\author{
Shinsuke Hara, Tatsuya Ishimoto, Masaya Kitano, and Tetsuo Tsujioka \\ Graduate School of Engineering, Osaka City University, Sumiyoshi-ku, Osaka 558-8585, Japan \\ Correspondence should be addressed to Shinsuke Hara, hara@info.eng.osaka-cu.ac.jp
}

Received 31 July 2008; Revised 30 December 2008; Accepted 18 February 2009

Recommended by Frank Ehlers

\begin{abstract}
In the motion control of a microrobot swarm, a key issue is how to autonomously generate a set of common coordinates among all robots and how to notify each robot of its heading direction in the generated common coordinates without any special devices for estimating location and bearing. This paper proposes a set of common coordinates and a heading direction generation method for a robot swarm with only received signal strength indicator (RSSI) measured through wireless communications. We explain the principle of the proposed method and show some computer simulation results on the location and direction estimation errors. Finally, we demonstrate some experimental results using a swarm composed of five robots with the IEEE 802.15.4 standard as its wireless communication tool.
\end{abstract}

Copyright ( 2009 Shinsuke Hara et al. This is an open access article distributed under the Creative Commons Attribution License, which permits unrestricted use, distribution, and reproduction in any medium, provided the original work is properly cited.

\section{Introduction}

A group of wirelessly networked robots is called a "robot swarm" $[1,2]$, and its promising applications include smart pills, drug delivery systems, and rescue systems. When a robot swarm is put into a new environment, member robots first start communicating with other (members) robots by wireless communication tool to recognize members of the swarm. Next, they try to understand their situations in the new environment by wireless communication, sharing and analyzing information obtained through their sensors. Finally, they decide and make a motion also by wireless communication to accomplish a given unified task as a group. Therefore, wireless communications play an important role in information transmission and motion control of robot swarm [3].

Especially for a microrobot swarm, since the size, energy, and memory of each robot are severely limited, functions of the swarm should be distributed over all robots; some robots are equipped with only the function of delivering energy to other robots, others are occupied with sensing the outside world and so on. The common function that all robots can have is wireless communications, so the functions imbeddable in wireless communications should be supported by the wireless communications. For example, ranging, namely, measuring the distance between a transmitter and a receiver, is easily supportable in wireless communications, so it should be supported by wireless communication protocol, without any special devices such as global positioning system (GPS) and geomagnetic sensors, which can be used to determine location and bearing.

Now, almost all of wireless communication standards, such as the IEEE 802.11 and 802.15.4 [4], support the function of measuring received signal power called "received signal strength indicator (RSSI)." This is because the RSSI can be measured by a very simple electronic circuit and its use for estimating wireless link quality effectively works. The RSSI drastically changes due to fading, shadowing, and near/far effect in wireless communications among robots because a signal emitted from a robot is reflected and shadowed by other robots [5]. However, once the medium of channel, types of transmitter/receiver antennas, type of carrier wave, and frequency/bandwidth of the carrier are given, we can derive the statistical model on the channel variation, namely, the relationship between the RSSI and distance, so a receiver can range for a transmitter with the RSSI. The advantage of RSSI ranging is that it is independent of the types of waveforms and that it is workable even in non-line-of-sight (NLOS) condition, although its accuracy is low. Therefore, in this paper, we assume an RSSI-based ranging with a 
prior knowledge on the relationship between the RSSI and distance.

There are mainly two methods of motion control for robot swarms, such as by ranging [6] and by localization [7]. The ranging-based motion control means that a leader robot decides and makes a motion, and other robots just follow it keeping the distance to it constant without knowing their locations and heading directions. On the other hand, the localization-based motion control means that all robots make their motions knowing their locations and heading directions in a set of common coordinates. In the localization-based motion control for a microrobot swarm, how to autonomously generate a set of common coordinates among robots and how to notify each robot of its heading direction in the coordinates without any special devices are key issues.

There have been many papers related to multirobot systems in the research fields of robotics and wireless communications. For the purpose of multirobot exploration and collaboration [8], several self-localization techniques have been proposed. For example, the tradeoff between localization efficiency and accuracy is discussed [9], and a Markov-based technique is proposed [10]. In these applications, to precisely control multirobots, intelligent devices are used, such as sonar, laser ranger finder, image sensor, and camera [11]. On the other hand, for mobile sensor networks, localization techniques without such intelligent devices are proposed [12, 13]. However, in these applications, GPS is often assumed for obtaining the locations of communication nodes, and the propagation characteristic of wireless signal is ignored.

In this paper, we propose a set of common coordinates and a heading direction generation method for a robot swarm with only RSSI-based ranging. Our key idea is based on the fact that when a robot receives a packet from another robot, it newly measures its RSSI related to the distance between them so the robot can improve its accuracy. In a fully networked robot swarm, our distributed localization method makes use of the effect, which has never been discussed in other literatures, so it can iteratively improve the location accuracy of each robot during their packet exchanging process. This accurate localization results in generating a set of accurate common coordinates thus accurate heading directions among robots.

In the research on swarm robotics, demonstration of the performance by experiments is important. Therefore, with commercially available wireless communication devices based on the IEEE 802.15.4 standard, we developed a swarm composed of 5 robots and conducted some experiments in indoor and outdoor environments. Here, to derive the localization algorithm, we took into consideration the propagation characteristic of the IEEE 802.15.4 signal. This algorithm is based on maximum likelihood estimation, which gives unbiased estimator [14].

The paper is organized as follows. Section 2 states the problem of common coordinates and heading direction generation and some assumptions to solve the problem. Section 3 presents the details of the proposed method, which is composed of three major components. Section 4 shows

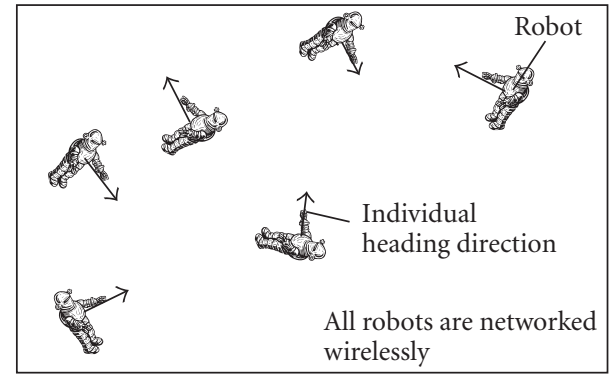

(a) Initial stage

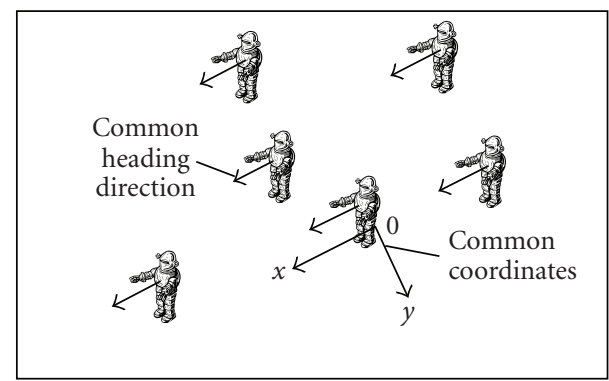

(b) Common coordinates/heading direction generation

Figure 1: Problem of common coordinates/heading direction generation.

some computer simulation results on the performance of the proposed method in terms of the location and direction estimation errors. Section 5 shows the experimental results using the swarm. Finally, Section 6 concludes the paper.

\section{Problem Statement of Common Coordinates/Heading Direction Generation}

Figure 1 shows the problem of the common coordinates and heading direction generation discussed in the paper.

In the initial stage, it is assumed that all robots have been wirelessly networked with each other, but that each robot has its own (different) individual heading direction (e.g., north) and no knowledge of its coordinates. The problem is generating a set of common coordinates among all of the robots and notifying each robot of its heading direction in the generated coordinates using only a ranging capability in wireless communications. Here, for the sake of simplicity, we assume that all of the robots are on the same plane, namely, we discuss a two-dimensional common coordinate generation: $(x, y)$. Note that the proposed method can be easily extended to the three-dimensional case.

A signal emitted from a robot experiences multipath reflections by other robots and surrounding obstacles, and furthermore, other robots in motion introduce a timevarying aspect to the signal received by each robot [5]. Therefore, the power (RSSI) of a received signal fluctuates in time. For the IEEE 802.15.4 signal, which has been adopted 
in our experiment, we can assume the following two-layered model on the distribution of the received power: $[15,16]$

$$
\begin{gathered}
\bar{P}=\alpha d^{-\beta}, \\
p(P \mid d)=\frac{1}{\bar{P}} \exp \left(-\frac{P}{\bar{P}}\right),
\end{gathered}
$$

where $P, \bar{P}$, and $d$ denote the received power, the average received power, and the distance between a transmitter robot and a receiver robot, respectively, and $p(P \mid d)$ denotes the conditional probability density function $(p d f)$ of $P$ when $d$ is given. In (1), $\alpha$ and $\beta$ are the constants that are uniquely determined by the medium of the channel and the carrier frequency and bandwidth of the signal. Note that the prerequisite knowledge on the channel parameters is not necessarily required, namely, they can be jointly estimated with the locations of robots [17].

\section{Proposed Common Coordinates/Heading Direction Generation Method}

The proposed common coordinates/heading direction generation method is composed of three elements, such as pivot robots selection, location estimation, and heading direction estimation.

3.1. Pivot Robots Selection. On a plane, if we know the locations of three different robots, we can uniquely determine the location of any robot according to these three locations. The proposed pivot robots selection chooses three robots with different locations as "pivot robots." Here, we assume that there are $M$ robots communicating with each other, and the robots are autonomously numbered at random as 1 to $M$ (ID number).

In the first step, each robot broadcasts $N_{o}$ "hello packets" containing its ID number to all other robots. Defining $P_{i j s}$ as the RSSI of the $s$ th packet $\left(s=1, \ldots, N_{o}\right)$ transmitted from the $i$ th robot and received at the $j$ th robot, with (1), the $j$ th robot can calculate the average RSSI and then the distance between them as $\left(j=1, \ldots, N_{o}, i \neq j\right)$

$$
\begin{gathered}
\bar{P}_{i j}=\frac{1}{N_{o}} \sum_{s=1}^{N_{o}} P_{i j s}, \\
d_{i j}=\left(\frac{\bar{P}_{i j}}{\alpha}\right)^{-1 / \beta},
\end{gathered}
$$

and broadcasts $d_{i j}$ to all other robots. In this way, all of the robots can share the information on the distances between all pairs of robots $d_{i j}(i, j=1, \ldots, M, i \neq j)$.

In the second step, each robot autonomously selects a pair of robots separated by the largest distance:

$$
\begin{gathered}
\text { select robots } i \text { and } j, \\
i, j=\underset{i, j}{\arg \max }\left\{d_{i j} \mid i, j=1, \ldots, M, i \neq j\right\},
\end{gathered}
$$

and each arbitrarily designates one of the two robots as the "master pivot robot", with the location vector of $\mathbf{Z}_{1}=$

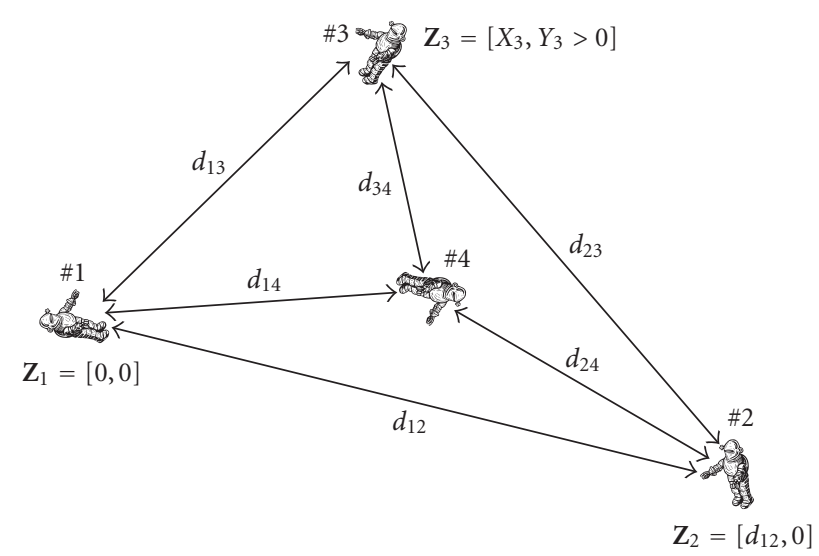

Figure 2: Pivot robots selection with $M=4$.

$\left[X_{1}, Y_{1}\right]=[0,0]$, whereas the other robot is designated as the "slave pivot robot", with the location of $\mathbf{Z}_{2}=\left[X_{2}, Y_{2}\right]=$ $\left[d_{i j}(>0), 0\right]$.

In the third step, as "another slave pivot robot," each robot autonomously selects a robot located farthest from the pivot robots selected in the second step:

$$
\begin{gathered}
\text { select robot } k, \\
k=\underset{k}{\arg \max }\left\{d_{i k}+d_{k j} \mid k=1, \ldots, M, k \neq i, j\right\},
\end{gathered}
$$

with the location vector of $Z_{3}=\left[X_{3}, Y_{3}>0\right]$ satisfying

$$
\begin{gathered}
X_{3}^{2}+Y_{3}^{2}=d_{i k}^{2}, \\
\left(d_{i j}-X_{3}\right)^{2}+Y_{3}^{2}=d_{k j}^{2} .
\end{gathered}
$$

In this way, each robot autonomously selects three pivot robots that are located far from each other. Finally, each robot then renumbers the master pivot robot as 1 . The slave pivot robots are renumbered as 2 and 3, and the other nonpivot robots are renumbered as 4 to $M$. Figure 2 shows an example of the (far) pivot robots selection with $M=4$ after the renumbering is finished.

Note that robots can randomly select three robots as one alternative and also they can select three robots located in close proximity to each other as another alternative. We will compare the location estimation performance among the far, random, and near pivot robots selections in Section 4.

3.2. Iterative Maximum Likelihood Location Estimation. Once the three pivot robots have been selected, they begin to broadcast their locations to all other robots. Here, we apply the index $l$ to the pivot robots $(l=1,2,3)$, whereas the index $m$ is applied to the nonpivot robots $(m=4, \ldots, M)$.

In the first step, each pivot robot broadcasts $N$ packets containing its ID number and location to all other robots. Defining the location vector of the $m$ th nonpivot robot as $\mathbf{z}_{m}=\left[x_{m}, y_{m}\right]$; the distance between the $l$ th pivot robot and the $m$ th nonpivot robot is written as

$$
d_{l m}=\left|\mathbf{Z}_{l}-\mathbf{z}_{m}\right|=\sqrt{\left(X_{l}-x_{m}\right)^{2}+\left(Y_{l}-y_{m}\right)^{2}} .
$$




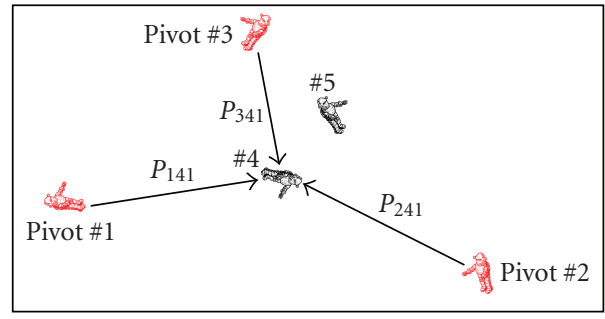

(a) First step for nonpivot robot \#4

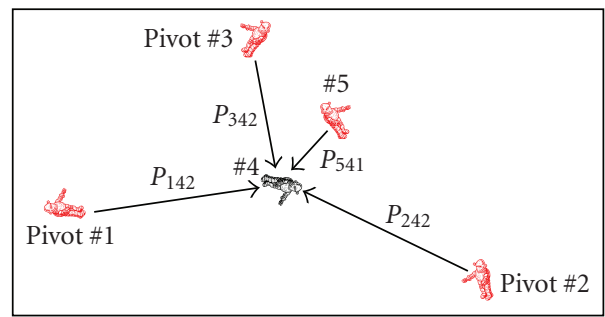

(c) Second step for nonpivot robot \#4

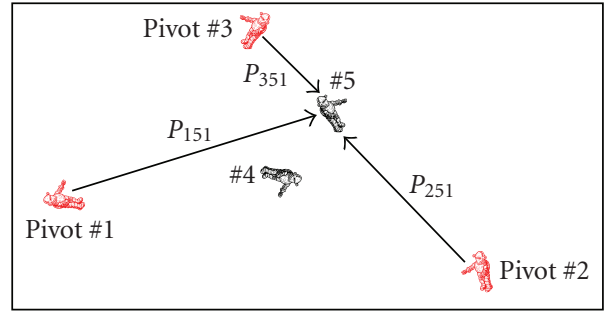

(b) First step for nonpivot robot \#5

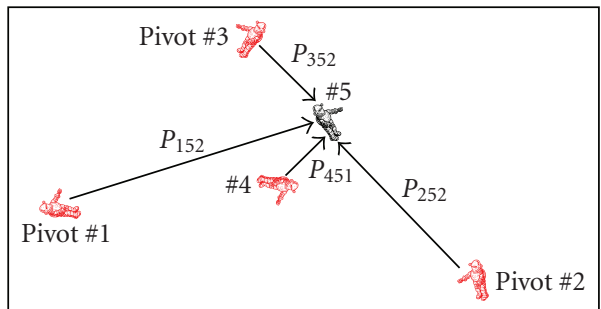

(d) Second step for nonpivot robot \#5

FIgURE 3: Iterative maximum likelihood location estimation with $M=5, N=1$, and $Q=1$.

Then, define the RSSI vector as

$$
\mathbf{P}_{m n}=\left[P_{1 m n}, P_{2 m n}, P_{3 m n}\right],
$$

where $P_{l m n}$ denotes the RSSI of the $n$th packet $(n=1, \ldots, N)$ transmitted from the $l$ th pivot robot and received at the $m$ th nonpivot robot. Since the unknown location of the nonpivot robot $\mathbf{z}_{m}$ is estimated with the measured RSSIs, the loglikelihood function on $\mathbf{z}_{m}$ is written with the conditional $p d f$ of $\mathbf{P}_{m n}(n=1, \ldots, N)$ when $\mathbf{z}_{m}$ is given as

$$
L\left(\mathbf{z}_{m}\right)=\log p\left(\mathbf{P}_{m 1}, \mathbf{P}_{m 2}, \ldots, \mathbf{P}_{m N} \mid \mathbf{z}_{m}\right) .
$$

Assuming that $P_{l m n}$ is statistically uncorrelated with $P_{l m n^{\prime}}$ $\left(n \neq n^{\prime}\right)$ (temporal whiteness) and $P_{l^{\prime} m n}\left(l \neq l^{\prime}\right)$ (geographical whiteness), replacing $d$ by $d_{l m}$ and $P$ by $P_{l m n}$, respectively, in (1) and (2), (10) yields

$$
\begin{aligned}
L\left(\mathbf{z}_{m}\right) & =\log \left[\prod_{n=1}^{N} \prod_{l=1}^{3}\left\{\frac{1}{\alpha d_{l m}^{-\beta}} \exp \left(\frac{-P_{l m n}}{\alpha d_{l m}{ }^{-\beta}}\right)\right\}\right] \\
& =N \sum_{l=1}^{3}\left\{\log \left(\frac{1}{\alpha d_{l m}{ }^{-\beta}}\right)-\frac{\sum_{n=1}^{N} P_{l m n} / N}{\alpha d_{l m}{ }^{-\beta}}\right\} .
\end{aligned}
$$

The ML estimation gives $\hat{\mathbf{z}}_{m 0}=\left[\hat{x}_{m 0}, \hat{y}_{m 0}\right]$, which maximizes (11) [14]

$$
\left.\frac{\partial L\left(\mathbf{z}_{m}\right)}{\partial \mathbf{z}_{m}}\right|_{\hat{\mathbf{z}}_{m 0}=\left[\hat{x}_{m 0}, \hat{y}_{m 0}\right]}=0 \quad(m=4, \ldots, M) .
$$

Since the locations of the nonpivot robots have been estimated in the first step, the robots also begin to broadcast their ID numbers and estimate locations to all other robots.
In the second step, each nonpivot robot estimates its location each time it receives broadcast packets from all other robots, and then broadcasts back a packet containing its newly estimated location with its ID number to all other robots. On the other hand, each pivot robot improves its location accuracy every time it receives broadcast packets from other pivot robots.

Define the estimated location vectors of the $l$ th pivot robot and the $m$ th nonpivot robot with the $q$ th broadcast packet as $\mathbf{Z}_{l q}$ and $\mathbf{z}_{m q}(q=1, \ldots, Q)$, respectively. $\mathbf{Z}_{l q}$ can be estimated by the same procedure in the pivot robots selection replacing $N_{o}$ by $q$ in (3). Here, the distance between the $l$ th pivot robot and the $m$ th nonpivot robot with the $q$ th broadcast packet is written as

$$
d_{l m q}=\left|\mathbf{Z}_{l q}-\mathbf{z}_{m q}\right| .
$$

On the other hand, when the $m$ th nonpivot robot receives the $q$ th broadcast packet from the $m^{\prime}$ th nonpivot robot with the RSSI of $P_{m^{\prime} m q}^{\prime}$, which contains the estimated location vector of the $m^{\prime}$ th nonpivot robot $\widehat{\mathbf{z}}_{m^{\prime}(q-1)}$, it can use the $m^{\prime}$ th nonpivot robot as a pivot robot with the location vector of $\hat{\mathbf{z}}_{m^{\prime}(q-1)}\left(m^{\prime}=4, \ldots, M, m^{\prime} \neq m\right)$. Namely, the distance between the $m$ th nonpivot robot and the $m^{\prime}$ th nonpivot robot with the temporarily known location vector of $\widehat{\mathbf{z}}_{m^{\prime}(q-1)}$ is

$$
\begin{aligned}
d_{m^{\prime} m q} & =\left|\hat{\mathbf{z}}_{m^{\prime}(q-1)}-\mathbf{z}_{m q}\right| \\
& =\sqrt{\left(\hat{x}_{m^{\prime}(q-1)}-x_{m q}\right)^{2}+\left(\hat{y}_{m^{\prime}(q-1)}-y_{m q}\right)^{2}},
\end{aligned}
$$


so the log-likelihood function on $\mathbf{z}_{m q}$ is written as

$$
\begin{aligned}
L\left(\mathbf{z}_{m q}\right)=\log \left[\prod_{q^{\prime}=1}^{q} \prod_{l=1}^{3}\left\{\frac{1}{\alpha d_{l m q^{\prime}}-\beta} \exp \left(\frac{-P_{l m q^{\prime}+N}}{\alpha d_{l m q^{\prime}}-\beta}\right)\right\}\right. \\
\left.\cdot \prod_{\substack{m^{\prime}=4 \\
m^{\prime} \neq m}}^{M}\left\{\frac{1}{\alpha d_{m^{\prime} m q^{\prime}}-\beta} \exp \left(\frac{-P_{m^{\prime} m q^{\prime}}}{\alpha d_{m^{\prime} m q^{\prime}}-\beta}\right)\right\}\right] \\
=\sum_{q^{\prime}=1}^{q}\left[\sum_{l=1}^{3}\left\{\log \left(\frac{1}{\alpha d_{l m q^{\prime}}-\beta}\right)-\frac{P_{l m q^{\prime}+N}}{\alpha d_{l m q^{\prime}}-\beta}\right\}\right. \\
\left.+\sum_{\substack{m^{\prime}=4 \\
m^{\prime} \neq m}}^{M}\left\{\log \left(\frac{1}{\alpha d_{m^{\prime} m q^{\prime}}}\right)-\frac{P_{m^{\prime} m q^{\prime}}}{\alpha d_{m^{\prime} m q^{\prime}}}\right\}\right] .
\end{aligned}
$$

The ML estimation yields $\hat{\mathbf{z}}_{m q}=\left[\hat{x}_{m q}, \hat{y}_{m q}\right]$, which maximizes (11)

$$
\left.\frac{\partial L\left(\mathbf{z}_{m q}\right)}{\partial \mathbf{z}_{m q}}\right|_{\hat{\mathbf{z}}_{m q}=\left[\hat{x}_{m q}, \hat{y}_{m q}\right]}=0 \quad(m=4, \ldots, M) .
$$

In this way, each nonpivot robot and each slave pivot robot can iteratively estimate their current locations with the previous locations of the other pivot robots and nonpivot robots up to $q=Q$. Figure 3 shows an example of iterative maximum likelihood location estimation with $M=5, N=$ 1 , and $Q=1$.

Note that all robots autonomously generate a set of common coordinates, so the coordinates have ambiguities such as translation, rotation, and negation with respect to the coordinates of an observer (operator) of the wirelessly networked robots. However, this is not critical because we can determine the relationship between the two coordinates.

3.3. Heading Direction Estimation. After the pivot robots selection and iterative maximum likelihood location estimation, all robots have generated a set of common coordinates, and each robot knows its location in the generated coordinates.

In the 0th step, the robots autonomously divide the set of all robots into $U$ subsets with equal numbers of robots. Next, in the $u$ th location/direction estimation step $(u=$ $1, \ldots, U)$ with $Q$ broadcast packets, each robot in the $u$ th subset moves through a distance of $B$, according to its own individual heading direction and stops, and its location is then estimated, starting with the robots in the other subsets $\left(u^{\prime}=1, \ldots, U, u^{\prime} \neq u\right)$ as pivot robots in the same manner as the iterative maximum likelihood location estimation. This process is repeated until $u=U$, and then the $m$ th robot $(m=1, \ldots, M)$ can determine its location before and after the movement, namely, $\mathbf{z}_{m}^{b}=\left[x_{m}^{b}, y_{m}^{b}\right]$ and $\mathbf{z}_{m}^{a}=\left[x_{m}^{a}, y_{m}^{a}\right]$. Finally, with the direction of the movement vector, the robot can estimate the angle between its heading direction and the $x$-axis in the generated coordinates, that is,

$$
\hat{\theta}_{m}=\arg \left(\mathbf{z}_{m}^{a}-\mathbf{z}_{m}^{b}\right) \text {. }
$$

Figure 4 shows an example of heading direction estimation with $M=6$ and $U=2$. Note that if a moving robot collides with another stationary robot, the moving robot returns to its original location, changes its heading direction by $+\gamma$ degrees, and moves again. This process is repeated until the robot has successfully finished moving through distance of $B$ without collision.

\section{Computer Simulation Results}

As shown in Section 5, we have developed a swarm composed of five robots to demonstrate the proposed common coordinates and heading direction generation method experimentally, where the PHY/MAC protocol is based on the IEEE 802.15.4 standard. Therefore, we determined the values of the two parameters in (1) as $\alpha=2.36 \times 10^{-6}$ and $\beta=2.37$ by a channel measurement experiment using a set of IEEE 802.15.4-based transceivers in a room.

In a computer simulation, we assume a field of $10 \mathrm{~m} \times 10 \mathrm{~m}$ and randomly select the locations of robots in the field. To speed up the process of generating the common coordinates and heading direction, we set $N_{o}=1$ and $N=1$. Furthermore, we refer to the number of broadcast packets (Q) as the "number of iterations."

Figure 5 shows the root mean square (RMS) location estimation error with respect to the number of iterations for the case of six robots. For all of the robots, as the number of iterations increases, the location estimation error gradually decreases because more packets (information) can be used for location estimation. For the pivot robots, the master robot is located at the origin, so its location estimation error is always zero, whereas the location estimation error of slave robot 3 is affected by that of slave robot 2, so the location estimation error of slave robot 3 is worse than that of slave robot 2. On the other hand, for the nonpivot robots, location estimation errors are affected by the worst location estimation error among the location estimation errors of the pivot robots. Therefore, the location estimation errors of the nonpivot robots are worse than the location estimation error of slave robot 3. However, there is no significant difference in the location estimation error among the nonpivot robots. In the following, the location estimation error is averaged over all types of robots such as master pivot, slave pivot, and nonpivot robots.

Figure 6 shows the effect of pivot robots selection for the case of six robots. The distances between pivot robots and nonpivot robots should be shorter because they have larger receiving powers and, consequently, smaller location estimation errors. In this sense, the case in which nonpivot robots are located in the area of a triangle formed by pivot robots as its three vertexes provides better location estimation performance. When three robots at distant locations from one another are selected as pivot robots, the triangle formed by the three pivot robots tends to include more nonpivot robots, so a smaller location estimation error is obtained, whereas when three robots in close proximity to one another are selected, a larger location estimation error is obtained. The performance provided by random robots 


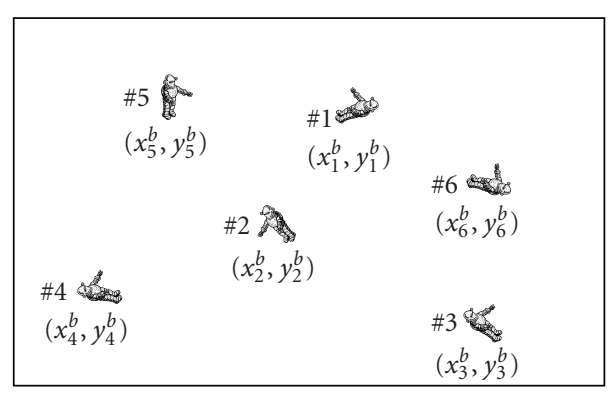

(a) 0th step

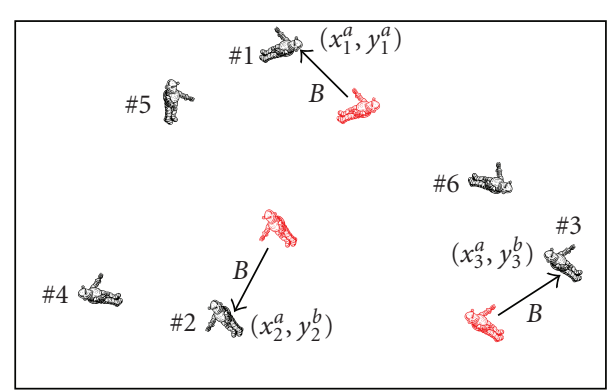

(b) First step

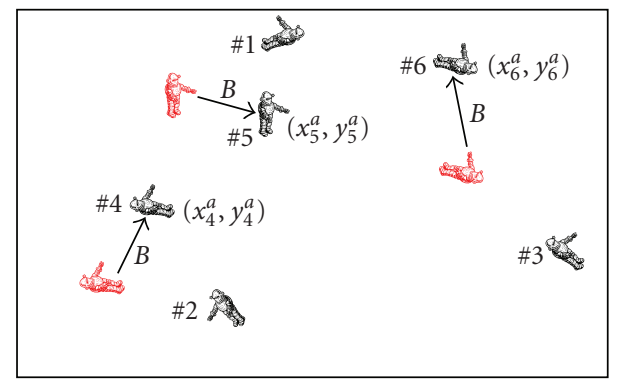

(c) Second step

FIgURE 4: Heading direction estimation with $M=6$ and $U=2$.

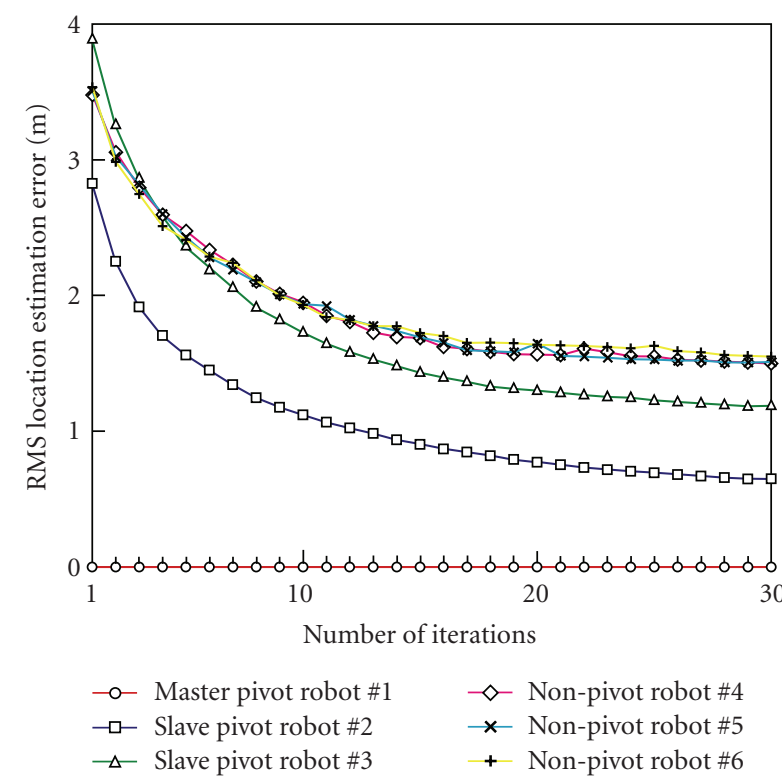

FIGURE 5: RMS location estimation error for individual robots.

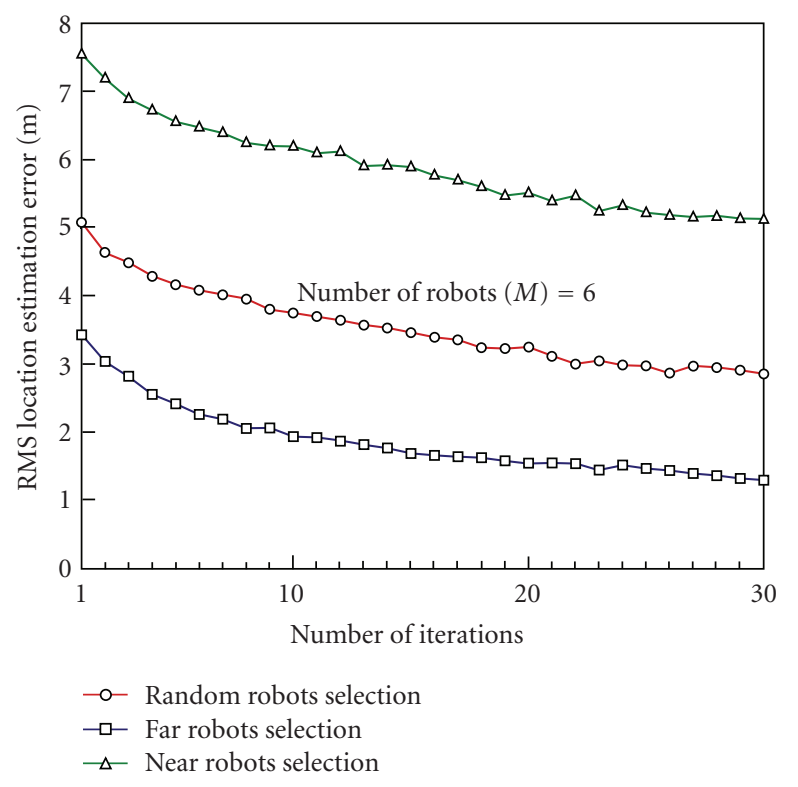

FIgURE 6: Effect of robots selection in location estimation. selection lies between those of the far and near robots selections.

Figure 7 shows the effect of the number of robots on the iterative location estimation performance. The iterative location estimation dealing with nonpivot robots as temporal pivot robots becomes more effective as the number of robots increases because packets from other nonpivot robots, even if they contain some ambiguities with respect to their locations, are helpful for improving the location estimation performance.

Figure 8 shows the RMS location estimation error in the heading direction estimation for the cases of $M=$ 20 and 30 with $U=2$ and $B=3 \mathrm{~m}$. Here, after the robots in the first location/heading direction estimation step move through $B=3$, their locations are estimated with 30 packets, and then the robots in the second step move. 


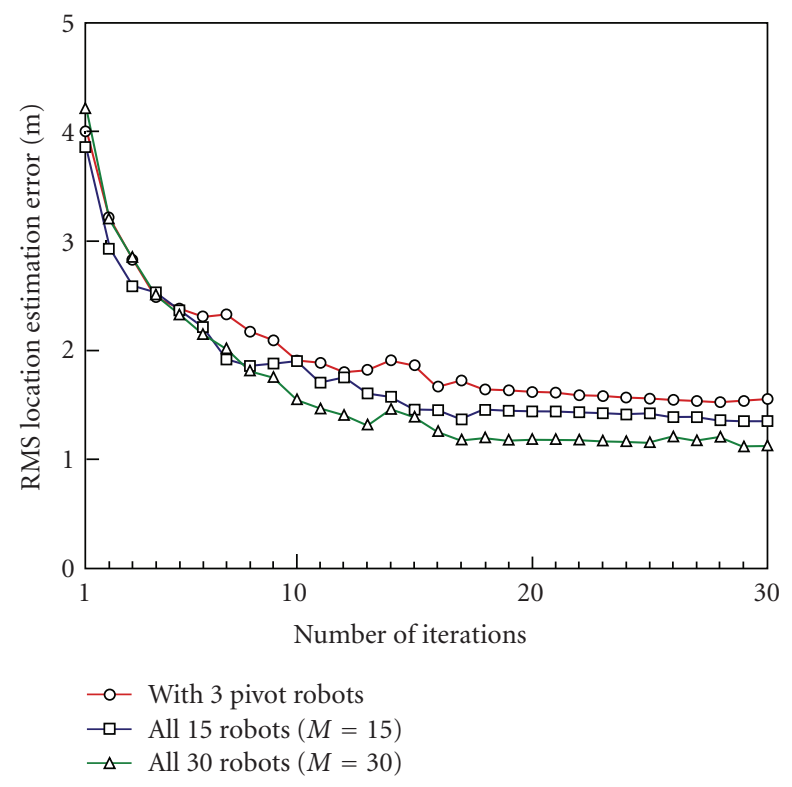

FIgURE 7: Effect of the number of robots in iterative location estimation.

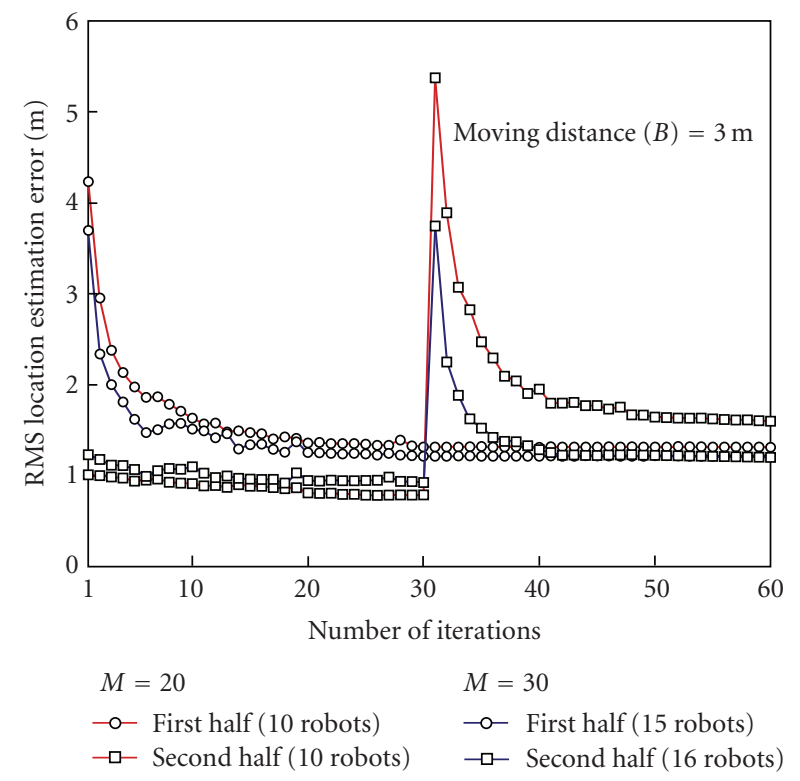

FIGURE 8: RMS location estimation error in the heading direction estimation.

At the beginning of the iterations in each location/direction estimation step, the location estimation error is large, but it is improved as the number of iterations increases. In addition, a larger total number of robots provides better location estimation performance. The robots in the first location/heading direction estimation step, with their poorer location estimation error, estimate the locations of the robots in the second step, so that the residual location estimation error in the second step is larger than that in the first step.

Figure 9 shows the effect of the number of location/heading direction estimation steps on the RMS location

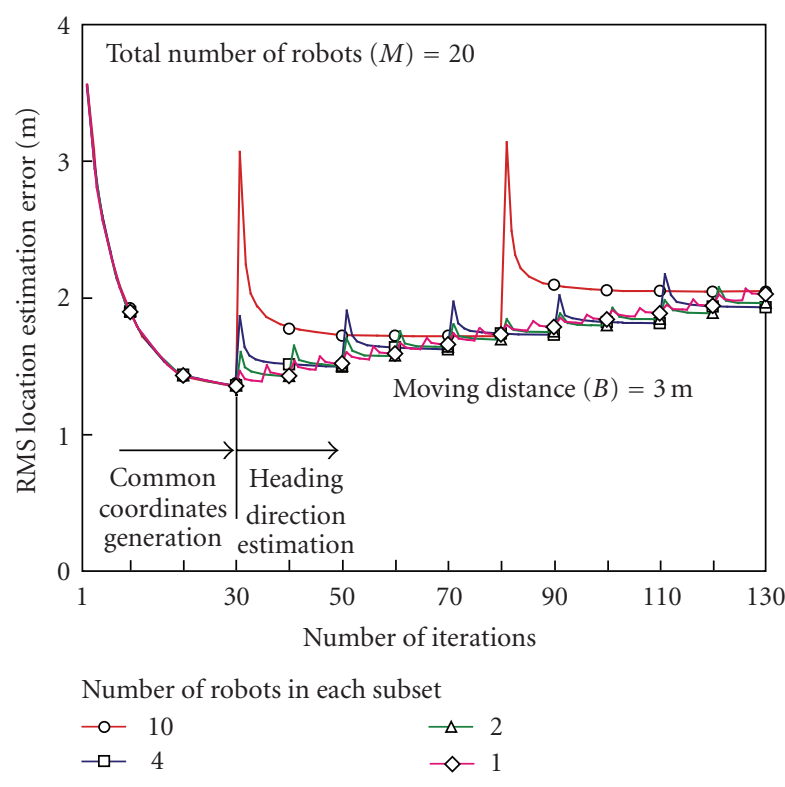

FIGURE 9: Effect of the number of location/direction estimation steps on the location estimation error in the heading direction estimation.

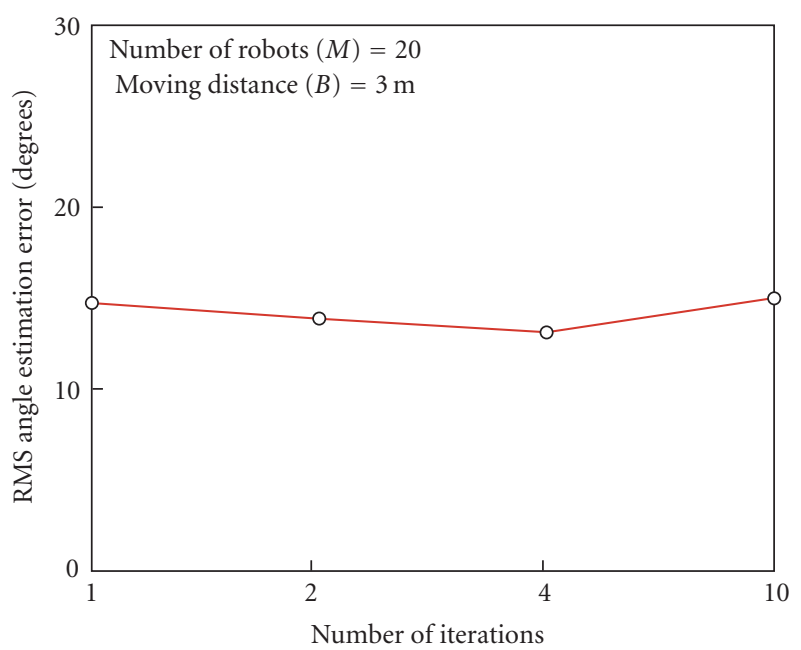

FIGURE 10: RMS location estimation error with respect to the number of location/direction estimation steps.

estimation error in the heading direction estimation for the case of $M=20$. The location estimation with the smaller number of robots in each subset (larger number of subsets) shows a quicker convergence. Therefore, in this case, the total number of iterations decreases as the number of robots in each subset decreases. Note that the location estimation error in a location/direction estimation step is affected by the residual location estimation errors in all of the location/direction estimation steps before the location/direction estimation step of interest. Therefore, the residual location estimation error is a monotonically increasing function on 


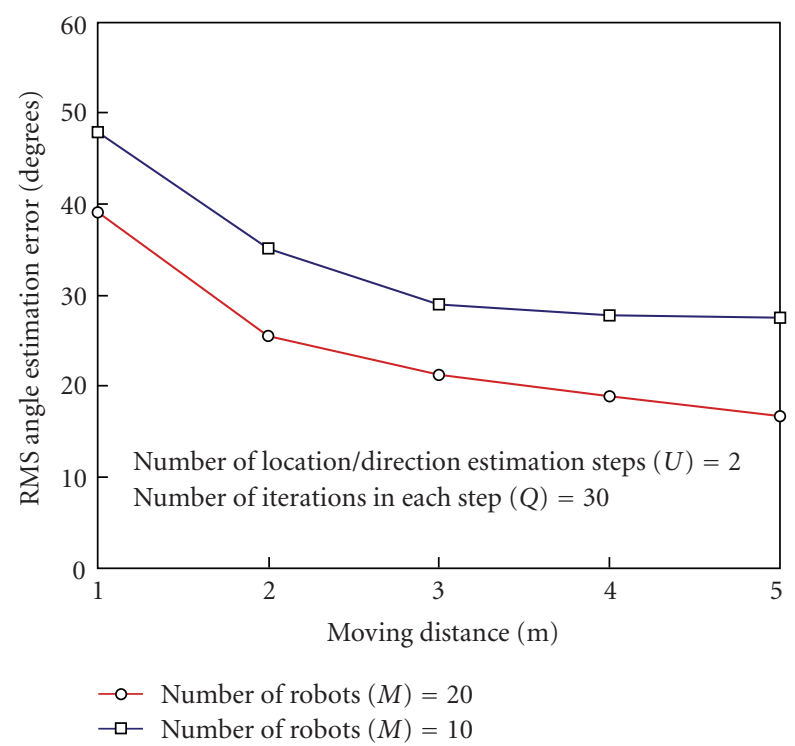

FIGURE 11: Effect of moving distance on heading direction estimation.

the index number of location/direction estimation steps. In this sense, fewer location/direction estimation steps provide better location estimation performance averaged over all robots. However, when the number of location/direction estimation steps is smaller, the residual location estimation error in each step is larger because the number of robots acting as pivots decreases. Therefore, for a given total number of robots, there is an optimal number of location/direction estimation steps that minimizes the location estimation error and, consequently, the heading direction estimation error averaged over all robots. Figure 10 shows the RMS angle estimation error with respect to the number of location/direction estimation steps. This figure clearly shows that, for 20 robots, the angle estimation error is minimized for the case of four steps.

Figure 11 shows the RMS angle estimation error with respect to the moving distance. In addition, Figure 12 shows the pdf of the angle estimation error. As the moving distance becomes larger, the RMS angle estimation error, namely, the standard deviation of the angle estimation error, decreases. However, "motion and its control" consume much more energy than "wireless communications." Therefore, consideration of the energy constraint in the problem of common coordinates and heading direction generation will be investigated in future studies.

Finally, Figure 13 shows an example of the obtained heading directions, where the arrows with solid and dashed lines show the real and estimated heading directions, respectively. With the RSSI-based location estimation, the performance of which is poorer than that of other methods, such as the TOA method, a set of common coordinates can be generated among all robots and each heading direction can be roughly estimated. Because of the poor performance of the RSSI-based location estimation, a fine direction estimation within a few degrees cannot be achieved. Therefore, in the next step to control all of the robots as a

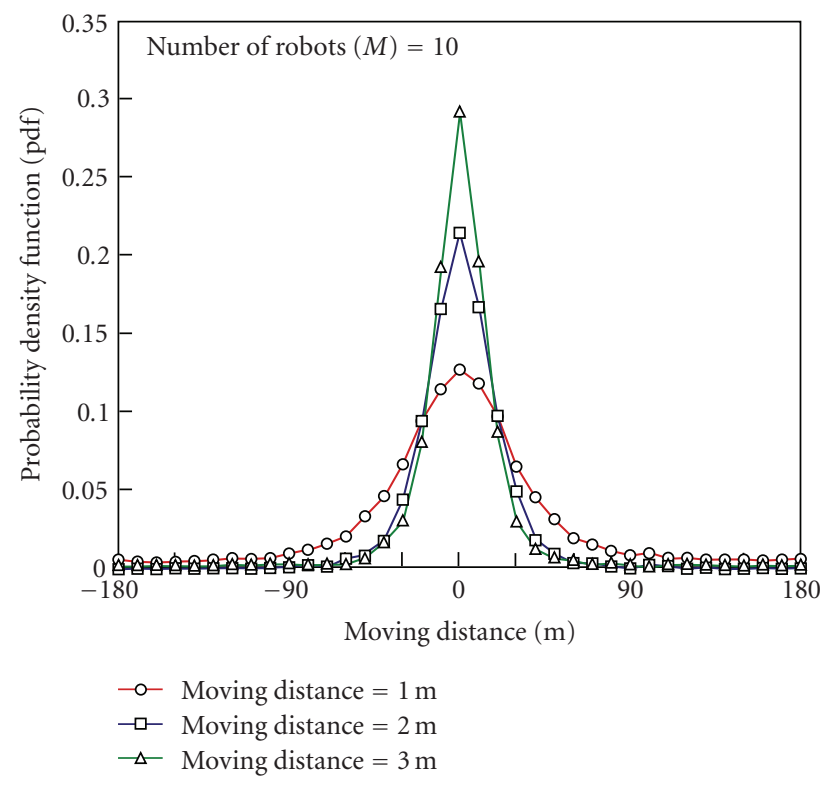

Figure 12: $P d f$ of the angle estimation error.

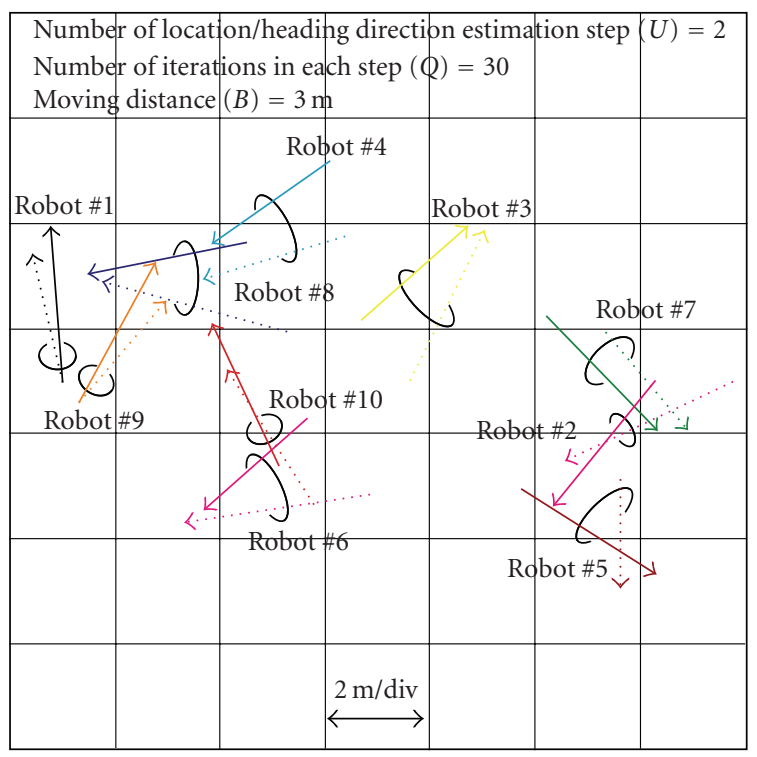

FIgURE 13: Example of estimated heading directions for 10 robots.

group in order to carry out a task, a method to generate a perfectly common heading direction is required.

\section{Experimental Results}

To evaluate the performance of the proposed common coordinates/heading direction estimation method experimentally, we have developed a swarm composed of 5 robots.

Figure 14 shows a photograph of a robot based on a tank kit manufactured by TAMIYA, and Figure 15 shows the inside of the robot, where the control element is composed of an I/O board, a CPU board, and a PHY/MAC board. The I/O board controls the DC motors of the robot for movement, 


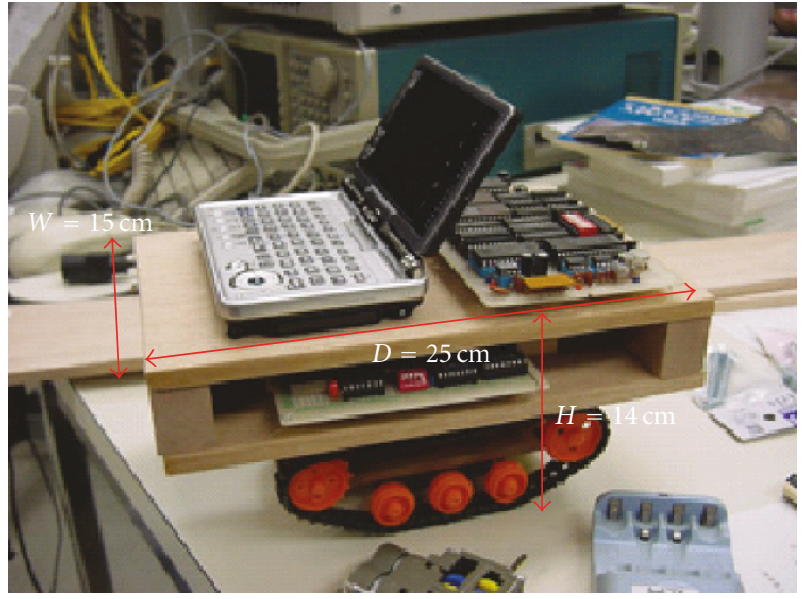

Figure 14: Photograph of a robot.

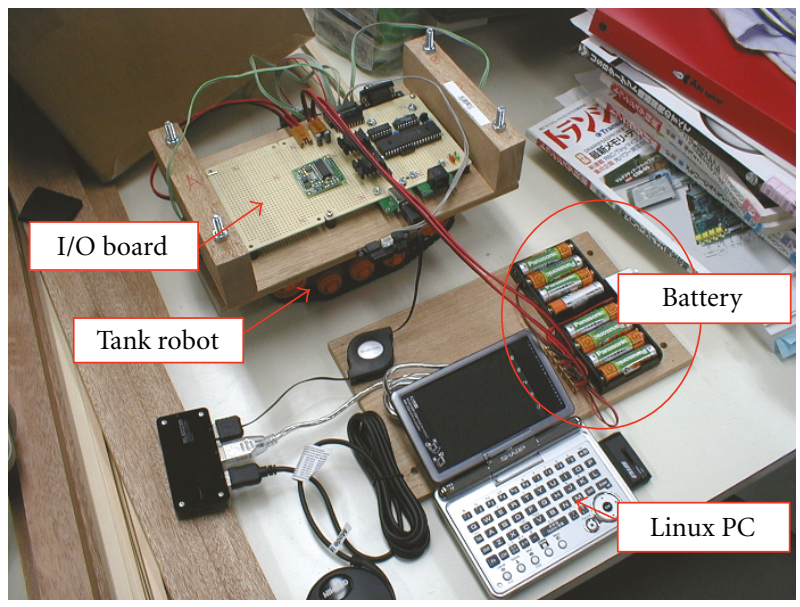

FIGURE 15: Photograph of the inside of the robot.

and optionally gathers information, such as temperature, from sensors. The CPU board, which is a Linux PC, is equipped with a high-speed processor $(416 \mathrm{MHz})$ and a sufficient memory (RAM: $64 \mathrm{MB}$, ROM: $128 \mathrm{MB}$ ) enabling both real-time wireless communications and motion control. The proposed common coordinates and heading direction generation algorithm can be programmed using $\mathrm{C}++$ via the PC. The Linux PC is also connected to an MICA-Z node supporting the IEEE 802.15.4 standard for wireless communications.

Figure 16 shows the I/O board in detail. The PIC interprets the commands from the $\mathrm{CPU}$ and drives the motors accordingly. In addition, the I/O board is equipped with an RS-232C port and a USB port, so that several sensors and input/output devices can be connected to the board.

We conducted experiments in two different environments. Figure 17 shows an outdoor environment which is a tennis court, whereas Figure 18 shows an indoor environment which is a lecture room with $W 6.96 \mathrm{~m} \times D 7.13 \mathrm{~m} \times H 2.61 \mathrm{~m}$. By premeasurements, we had $\alpha=9.1 \times 10^{-8}$ and $\beta=3.0$ for the outdoor

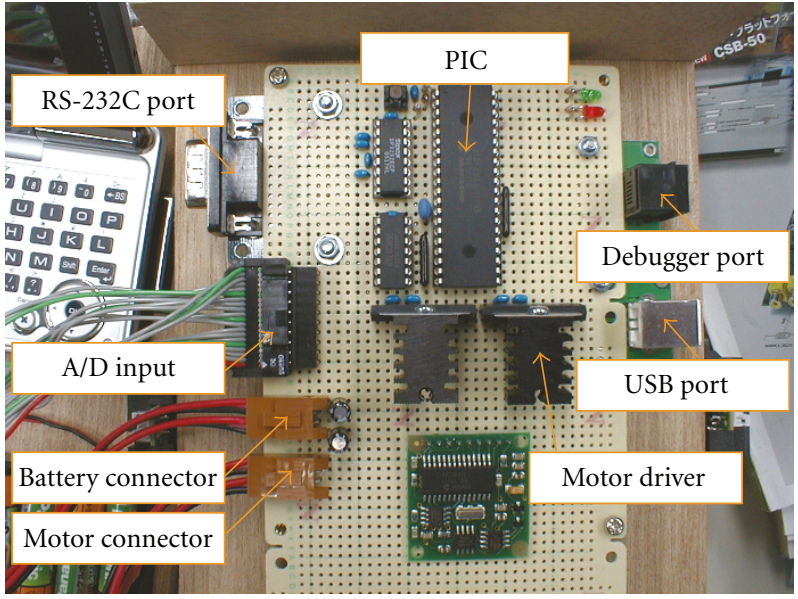

Figure 16: Detail of the I/O board.

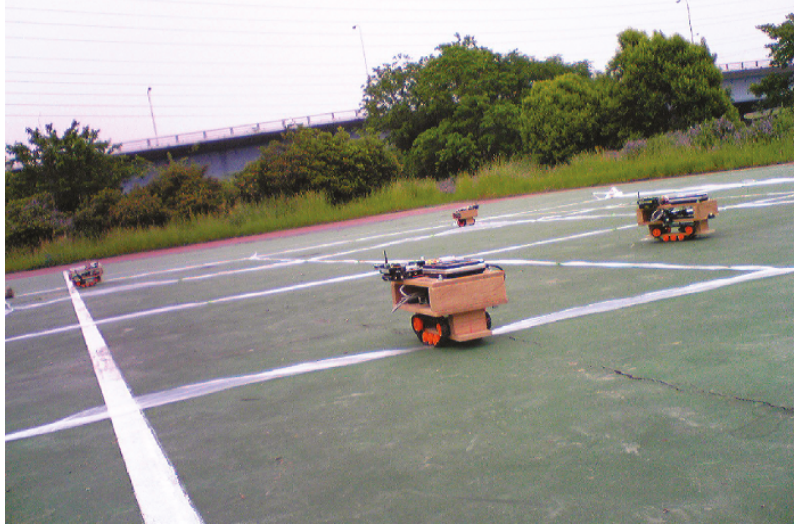

Figure 17: Photograph of an outdoor experiment.

environment and $\alpha=6.0 \times 10^{-7}$ and $\beta=2.5$ for the indoor environment. Note that even in the outdoor environment, the variation of the received power was observed due to reflection by the ground.

Figure 19 shows the experimental result on the root mean square (RMS) location estimation error. The RMS location estimation error is insensitive to the moving distance and it ranges around in $2.0 \mathrm{~m}$ to $3.0 \mathrm{~m}$. There is no large difference in the RMS location estimation error between the outdoor and indoor environments.

Figure 20 shows the experimental result on the RMS angle estimation error. Although the RMS location estimation error is insensitive to the moving distance, the RMS angle estimation error is sensitive to the moving distance, namely, the RMS angle estimation error decreases as the moving distance increases. This is because the angle between the departure point and the arrival point of a robot, which is observed and thus estimated by another stationary robot, is in proportional to the moving distance. There is a little difference in the RMS angle estimation error between the outdoor and indoor environments. This is because (2) is 


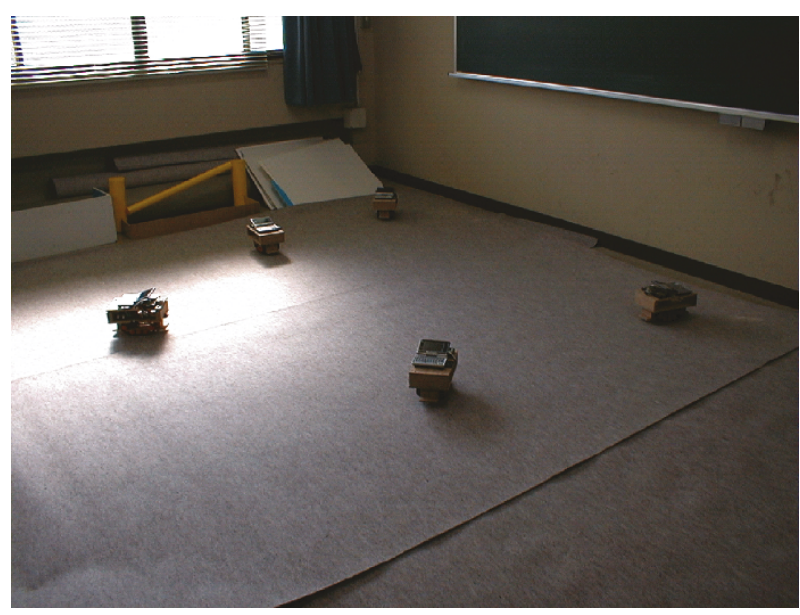

FIgURE 18: Photograph of an indoor experiment.

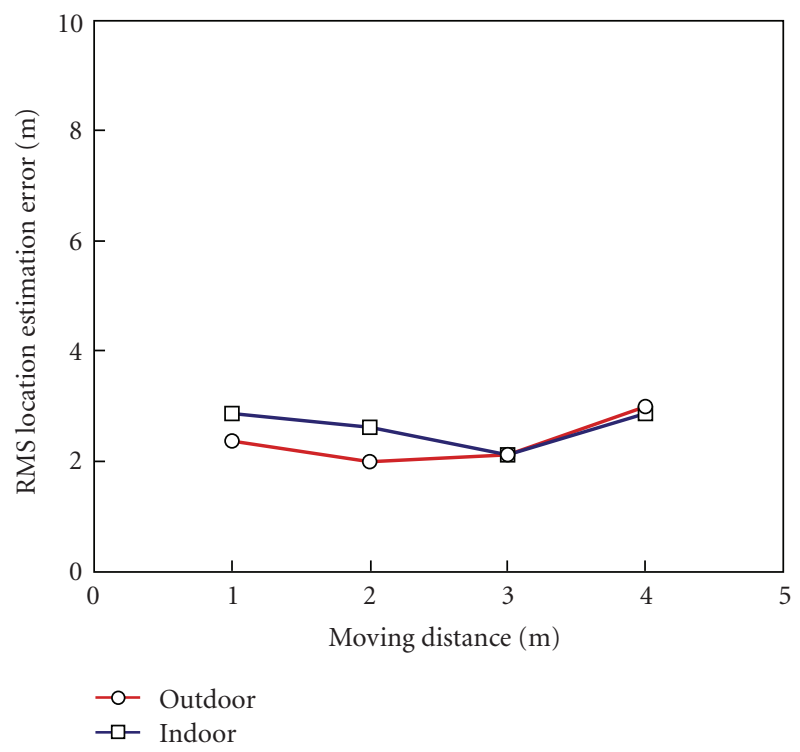

FIGURE 19: Experimental result on the RMS location estimation error.

valid only for the scattering (multipath)-rich condition not in the outdoor environment but in the indoor environment. For the case of the indoor environment, the RMS angle error of around 40 degrees is obtained, which is enough for coarse motion control of each robot.

\section{Conclusions}

This paper has proposed a set of common coordinates and a heading direction generation method for a robot swarm with only ranging. We have assumed an RSSI measurement as a ranging method, which is easily realized in wireless communications (however, it is not the only ranging method available to us). By computer simulations, we have revealed the following.

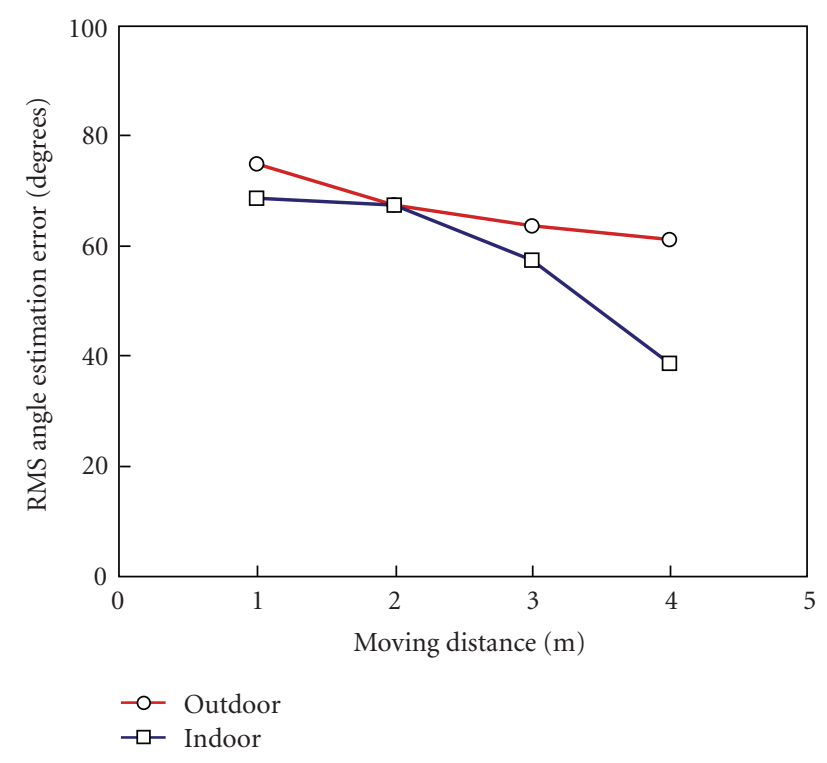

FIGURE 20: Experimental result on the RMS angle estimation error.

(i) Without any known locations of robots, a set of common coordinates can be autonomously generated in a robot swarm with only an RSSI-based ranging in wireless communication tool.

(ii) The far robots selection outperforms the random and near robots selections in terms of location estimation accuracy thus heading direction accuracy.

(iii) The iterative location estimation method effectively improves the accuracy.

(iv) The location and angle estimation accuracies improve as the number of robots increases, and the angle estimation accuracy also improves as the moving distance increases.

We have taken into consideration the large variation of the received signal power resulting from multipath fading as well as the near/far effect even in the computer simulation, so the proposed method can achieve an angle estimation error from the $x$-axis of approximately 18 degrees for the case of 20 robots, 30 iterations, two location/direction estimation steps, and a moving distance of $5 \mathrm{~m}$.

On the other hand, in the experiments with a swarm composed of five robots, we have demonstrated the location and angle estimation errors in outdoor and indoor environments. Because of the limited number of robots, a lowangle estimation accuracy of 40 degrees has been obtained for a moving distance of $5 \mathrm{~m}$ in the indoor environment. This value is enough for the coarse angle estimation in the initial stage of the motion control of the robot swarm. An additional heading direction generation method is required that can achieve fine angle estimation after the coarse angle estimation is achieved using the proposed method. We intend to investigate such a method in the future.

Finally, the proposed method is based on the maximum likelihood estimation with a nonlinear function shown in 
(15), so that the computational complexity is high. If the conditional $p d f$ of the distance can be approximated with a Gaussian function, we can use a distributed belief propagation method [18]. Furthermore, even in general form, we can use a distributed particle filter method [19].

\section{Acknowledgment}

This study was supported in part by a Grant-in-Aid for Scientific Research (no. 19360177) from the Ministry of Education, Science, Sport and Culture of Japan.

\section{References}

[1] J.-P. Hubaux, T. Gross, J.-Y. Le Boudec, and M. Vetterli, "Toward self-organized mobile ad hoc networks: the terminodes project," IEEE Communications Magazine, vol. 39, no. 1, pp. 118-124, 2001.

[2] Z. Butler and D. Rus, "Event-based motion control for mobilesensor networks," IEEE Pervasive Computing, vol. 2, no. 4, pp. 34-42, 2003.

[3] S. Hara, H. Yomo, P. Popovski, and K. Hayashi, "New paradigms in wireless communication systems," Wireless Personal Communications, vol. 37, no. 3-4, pp. 233-241, 2006.

[4] IEEE Std.802.15.4, "Wireless Medium Access Control (MAC) and Physical Layer (PHY) Specifications for High Layer Wireless Personal Area Networks (WPANs)," 2003.

[5] W. C. Y. Lee, Mobile Communications Engineering, McGrawHill, New York, NY, USA, 1982.

[6] S. Hara, M. Kitano, and T. Ishimoto, "AMEBA: autonomous enmeshing and banding algorithm for wireless-networked robots," in Proceedings of the International Symposium on Wireless Personal Multimedia Communications (WPMC '08), pp. 711-714, Jaipur, India, December 2007, CD-ROM.

[7] S. Hara and T. Ishimoto, "A common coordinates/heading direction generation method for wirelessly networked robots with only ranging capability," in Proceedings of the 1st International Conference on Robot Communication and Coordination (ROBOCOMM '07), pp. 1-36, Athens, Greece, October 2007, CD-ROM.

[8] I. M. Rekleitis, G. Dudek, and E. E. Milios, "Multi-robot collaboration for robust exploration," in Proceedings of IEEE International Conference on Robotics and Automation (ICRA '00), vol. 4, pp. 3164-3169, San Francisco, Calif, USA, April 2000.

[9] I. M. Rekleitis, G. Dudek, and E. E. Milios, "Multi-robot cooperative localization: a study of trade-offs between efficiency and accuracy," in Proceedings of the IEEE/RSJ International Conference on Intelligent Robots and Systems (IRDS '02), vol. 3, pp. 2690-2695, Lausanne, Switzerland, September-October 2002.

[10] D. Fox, W. Burgard, and S. Thrun, "Active Markov localization for mobile robots," Robotics and Autonomous Systems, vol. 25, no. 3-4, pp. 195-207, 1998.

[11] J. R. Spletzer, Sensor fusion techniques for cooperative localization in robot teams, Ph.D. thesis, University of Pennsylvania, Philadelphia, Pa, USA, 2003.

[12] C.-H. Wu, W. Sheng, and Y. Zhang, "Mobile sensor networks self localization based on multi-dimensional scaling," in Proceedings of the IEEE International Conference on Robotics and Automation (ICRA '07), pp. 4038-4043, Rome, Italy, April 2007.
[13] S. Tilak, V. Kolar, N. B. Abu-Ghazaleh, and K.-D. Kang, "Dynamic localization control for mobile sensor networks," in Proceedings of the 24th IEEE International Performance, Computing, and Communications Conference (IPCCC '05), pp. 587-592, Phoenix, Ariz, USA, April 2005.

[14] N. Patwari, A. O. Hero III, M. Perkins, N. S. Correal, and R. J. O'Dea, "Relative location estimation in wireless sensor networks," IEEE Transactions on Signal Processing, vol. 51, no. 8, pp. 2137-2148, 2003.

[15] S. Hara, D. Zhao, K. Yanagihara, et al., "Propagation characteristics of IEEE 802.15.4 radio signal and their application for location estimation," in Proceedings of the 61th IEEE Vehicular Technology Conference (VTC'05), vol. 1, pp. 97-101, Stockholm, Sweden, May 2005.

[16] R. Zemek, D. Zhao, M. Takashima, et al., "A traffic reducing method for multiple targets localisation in an IEEE 802.15.4 based sensor network," in Proceedings of the 64th IEEE Vehicular Technology Conference (VTC '06), pp. 2499-2503, Montreal, Canada, September 2006, CD-ROM.

[17] R. Zemek, S. Hara, K. Yanagihara, and K.-I. Kitayama, "A joint estimation of target location and channel model parameters in an IEEE 802.15.4-based wireless sensor network," in Proceedings of the 18th Annual IEEE International Symposium on Personal, Indoor and Mobile Radio Communications (PIMRC '07), pp. 1-5, Athens, Greece, September 2007, CDROM.

[18] D. Koller, U. Lerner, and D. Angelov, "A general algorithm for approximate inference and its application to hybrid bays nets," in Proceedings of the 15th Conference on Uncertainty in Artificial Intelligence (UAI '99), pp. 324-333, Stockholm, Sweden, JulyAugust 1999.

[19] A. T. Ihler, J. W. Fisher III, R. L. Moses, and A. S. Willsky, "Nonparametric belief propagation for self-localization of sensor networks," IEEE Journal on Selected Areas in Communications, vol. 23, no. 4, pp. 809-819, 2005. 